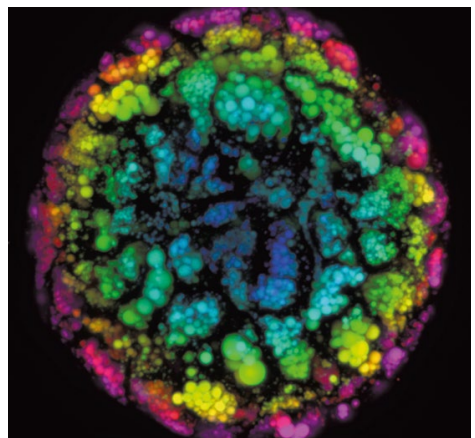

Image courtesy of T. Takebe, Cincinnati Children's Hospital Medical Center, USA.

and Kupffer-like cells released proinflammatory cytokines. Stellate-like cells displayed markers of activation, and levels of collagen were markedly increased in FA-treated HLOs.

The authors hope that further refinement of their model will enable the investigation of human genetic factors influencing NAFLD development. "As a next step, coupled with our unique organoid steatohepatitis model, we plan to investigate genetic variations for studying personalized NAFLD mechanisms that reflect clinical variations found in vivo," says Takebe.

Hugh Thomas

ORIGINAL ARTICLE Ouchi, R. et al. Modeling steatohepatitis in humans with pluripotent stem cell-derived organoids. Cell Metab. https://doi.org/ 10.1016/j.cmet.2019.05.007 (2019)

T cells and the HBV entry inhibitor myrcludex $B$ led to long-term virological control, presumably by preventing reinfection of remaining hepatocytes following the inevitable clearance of the human T cells.

Overall, the study provides preclinical evidence that TCR-grafted $T$ cells can restore natural $T$ cell immunity and could achieve functional cure in patients with $\mathrm{CHB}$, or even patients with $\mathrm{HBV}$-associated hepatocellular carcinoma (HCC) expressing HBV antigens.

"Together with the German Research Center for Environmental Health (HMGU), we are now focusing on preparing a clinical trial for treating patients with $\mathrm{HBV}$-associated HCC with T cell therapy," concludes author Ulrike Protzer.

Conor A. Bradley
TCR-grafted

T cells can restore natural T cell immunity

\section{HAstening the development of liver fibrosis}

New experimental research has demonstrated that hyaluronan (HA), a component of the extracellular matrix, is involved in the development of liver fibrosis, with hyaluronan synthase 2 (HAS2) having a central role in the production of $\mathrm{HA}$, the activation of hepatic stellate cells (HSCs) and the progression of liver fibrosis.

The production and deposition of ECM is a prominent feature of liver fibrosis, with HSC activation established as a central driver of fibrosis during liver injury. "HA has been known as a biomarker for cirrhosis. However, whether HA plays a functional role in the development of liver fibrosis (or is just a by-stander) was unknown," says author Ekihiro Seki.

The researchers first examined liver biopsy samples and identified that HAS2 and HA were overexpressed in human liver fibrosis of differing aetiologies (induced by either hepatitis $\mathrm{B}$, hepatitis $\mathrm{C}$ or NASH). This finding was recapitulated in several mouse models of liver fibrosis, including cholestasis-induced, NASH-induced and toxin-induced fibrosis models. Crucially, HAS2 was confirmed as the critical enzyme for HA production and liver fibrosis in mouse models. In mice lacking Has2 in HSCs, both HA and liver fibrosis levels were reduced; conversely, mice overexpressing Has2 overproduced HA, promoting HSC activation and exacerbating liver fibrosis.

Further experiments examined changes in transcriptional regulation downstream from HAS2 expression and identified $\mathrm{NOTCH} 1$ as an important effector of the HAS2-HA-CD44 signalling that promoted liver fibrosis. Importantly, pharmacological inhibition of HA synthesis with 4-methylumbelliferone (4-MU, a drug used for the treatment of biliary spasm) reduced HSC activation and prevented liver fibrosis progression in a mouse model of liver injury.

"In the maintenance of systemic levels of circulating HA, the balance between production and clearance is important," notes Seki, with liver sinusoidal endothelial cells (LSECs) having a central role in the elimination of HA. "Because LSECs are dysfunctional in liver fibrosis and cirrhosis, HA cannot be eliminated properly; however, the production mechanism of HA in liver fibrosis and cirrhosis had not been tested," he adds. "As blocking HA synthesis by 4-MU suppressed liver fibrosis, we should think about targeting this pathway for a treatment option for liver fibrosis - either to use 4-MU or 4-MU derivatives, or to develop new compounds to block HAS2 activity," explains Seki, highlighting that, as yet, there are no effective anti-fibrotic drugs available for liver fibrosis.

Katrina Ray

ORIGINAL ARTICLE Yang, Y. M. et al. Hyaluronan synthase 2-mediated hyaluronan production mediates Notch1 activation and liver fibrosis. Sci. Transl Med. 11, eaat9284 (2019)

FURTHER READING Tsuchida, T. \& Friedman, S. L. Mechanisms of hepatic stellate cell activation. Nat. Rev. Gastroenterol. Hepatol. 14, 397-411 (2017)

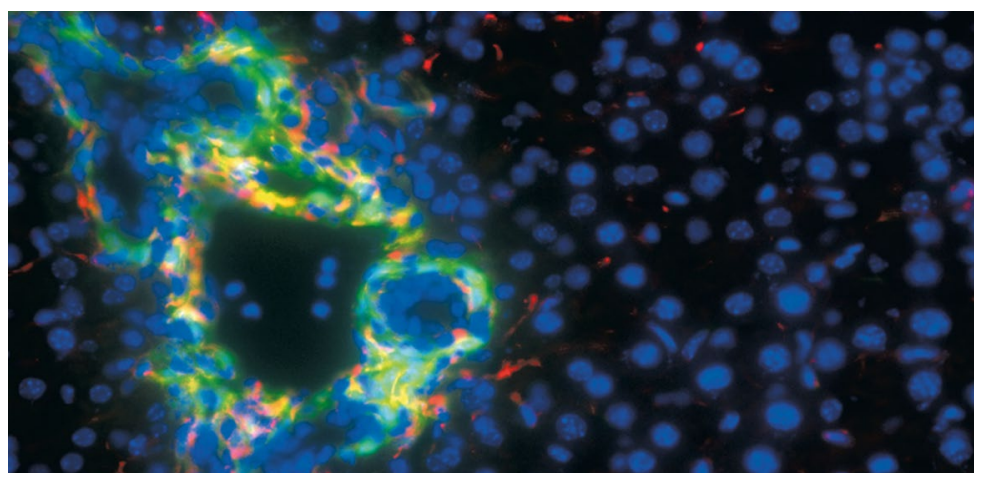

Hyaluronan expression during liver fibrosis in mice (green, hyaluronan; red, desmin). Image courtesy of E. Seki, Cedars-Sinai Medical Center, USA. 